\title{
FREQUENCY OF OBSESSIVE AND COMPULSIVE SYMPTOMS IN PATIENTS WITH BLEPHAROSPASM AND HEMIFACIAL SPASM
}

\author{
R.P. Munhoz, H.A.G. Teive, M.V. DellaColletta, F.M.B. Germiniani, F.M. Iwamoto, C.H.F. Camargo, L.C. Wemeck
}

Arq Neuropsiquiatr 2005; 63:213-216

Tables of this article are missing*. They appear below.

\begin{tabular}{|c|c|c|c|c|c|c|c|}
\hline Symptoms & $\mathrm{N}^{\circ}$ & Mean & $\begin{array}{l}\text { Standard } \\
\text { deviation }\end{array}$ & Minimum & Maximum & Median & $\mathrm{p}^{(1)}$ \\
\hline \multicolumn{8}{|l|}{ Obsessive } \\
\hline BS & 13 & 5,9 & 3,8 & 1,0 & 11,0 & 7,0 & \multirow[b]{2}{*}{0,201} \\
\hline HFS & 08 & 3,6 & 1,8 & 1,0 & 6,0 & 4,0 & \\
\hline \multicolumn{8}{|l|}{ Compulsive } \\
\hline BS & 03 & 12,7 & 5,5 & 9,0 & 19,0 & 10,0 & \multirow[b]{2}{*}{-} \\
\hline HFS & 04 & 4,8 & 4,1 & 1,0 & 10,0 & 4,0 & \\
\hline \multicolumn{8}{|l|}{ Both } \\
\hline BS & 04 & 14,0 & 14,2 & 4,0 & 35,0 & 8,5 & \multirow{2}{*}{0,938} \\
\hline HFS & 09 & 9,4 & 3,5 & 4,0 & 15,0 & 9,0 & \\
\hline \multicolumn{8}{|l|}{ Total } \\
\hline BS & 20 & 8,6 & 7,6 & 1,0 & 35,0 & 7,5 & \multirow[b]{2}{*}{0,417} \\
\hline HFS & 21 & 6,3 & 4,0 & 1,0 & 15,0 & 6,0 & \\
\hline
\end{tabular}

Table 2. Patients with concomitant obsessive and compulsive symptoms with corresponding Yale-Brown scale scores.

\begin{tabular}{ccccc}
\hline Patient & MD & Obsessive symptoms & Compulsive symptoms & Total \\
\hline 1 & BS & 3 & 1 & 4 \\
2 & BS & 3 & 4 & 7 \\
3 & BS & 18 & 17 & 33 \\
4 & BS & 3 & 7 & 10 \\
5 & HFS & 7 & 5 & 12 \\
6 & HFS & 3 & 1 & 4 \\
7 & HFS & 6 & 3 & 9 \\
8 & HFS & 9 & 3 & 12 \\
9 & HFS & 10 & 4 & 14 \\
10 & HFS & 11 & 2 & 13 \\
11 & HFS & 7 & 4 & 11 \\
12 & HFS & 2 & 4 & 6 \\
\hline
\end{tabular}

MD, movement disorder; BS, blepharospasm; HFS, hemifacial spasm.

*The Editor presents to the Authors his excuses for such omission. 